\title{
CAMERON-MARTIN TRANSLATION THEOREMS IN THE WIENER SPACE OF FUNCTIONS OF TWO VARIABLES $\left({ }^{1}\right)$
}

\author{
BY \\ J. YEH
}

1. Introduction. The Cameron-Martin translation theorems [1] have proved useful in the evaluation of various Wiener integrals. The analogue of one of these theorems for the integration of continuous functionals in the Wiener space of functions of two variables was considered by Kitagawa [2]. The present author has proved that the Wiener measure in the space of functions of two variables is indeed a measure [3]. In this article we give a rigorous statement together with a proof for the theorem of Kitagawa and then prove the translation theorem for Wiener measurable sets and Wiener integrals of arbitrary measurable functionals.

The Wiener space $C$ of functions of two variables is the collection of real valued continuous functions $f(x, y)$ defined on the unit square $Q: 0 \leqq x, y \leqq 1$ satisfying $f(0, y)=f(x, 0)=0$. A subset $I$ of $C$ defined by

$$
I\left\{x_{1}, \cdots, x_{m}, y_{1}, \cdots, y_{n}, E\right\}=\left\{f \in C ;\left[f\left(x_{1}, y_{1}\right), \cdots, f\left(x_{m}, y_{n}\right)\right] \in E\right\}
$$

where $0=x_{0}<x_{1}<\cdots<x_{m} \leqq 1, \quad 0=y_{0}<y_{1}<\cdots<y_{n} \leqq 1$ and $E$ is a Lebesgue measurable set in the $m n$-dimensional Euclidean space $R_{m n}$ is called an interval in $C$. The points $\left(x_{1}, y_{1}\right), \cdots,\left(x_{m}, y_{n}\right)$ are called the restriction points and the set $E$ the restricting set of $I$. The collection $\mathfrak{I}$ of all such intervals constitutes an interval class.

If we define for every $I \in \mathfrak{I}$

$$
\begin{aligned}
& m(I)=K\left\{x_{1}, \cdots, x_{m}, y_{1}, \cdots, y_{n}\right\} \\
& \cdot \int\left(m_{E} n\right) \int W\left\{x_{1}, \cdots, x_{m}, y_{1}, \cdots, y_{n}, u_{11}, \cdots, u_{m n}\right\} d u_{11}, \cdots, d u_{m n}
\end{aligned}
$$

where

$$
\begin{aligned}
& K\left\{x_{1}, \cdots, x_{m}, y_{1}, \cdots, y_{n}\right\} \\
& =\frac{1}{\left\{\pi^{m n}\left[x_{1}\left(x_{2}-x_{1}\right) \cdots\left(x_{m}-x_{m-1}\right)\right]^{n}\left[y_{1}\left(y_{2}-y_{1}\right) \cdots\left(y_{n}-y_{n-1}\right)\right]^{m}\right\}^{1 / 2}}
\end{aligned}
$$

Received by the editors August 4, 1961 and, in revised form, June 11, 1962.

(1) This research was supported in part by the United States Air Force, through the Office of Scientific Research of the Air Research and Development Command under the contract No. AF49(638)-1046. 


$$
\begin{aligned}
& W\left\{x_{1}, \cdots, x_{m}, y_{1}, \cdots, y_{n}, u_{11}, \cdots, u_{m n}\right\} \\
& \quad=\exp \left\{-\sum_{i=1}^{m} \sum_{j=1}^{n} \frac{\left(u_{i j}-u_{i, j-1}-u_{i-1, j}+u_{i-1, j-1}\right)^{2}}{\left(x_{i}-x_{i-1}\right)\left(y_{j}-y_{j-1}\right)}\right\}
\end{aligned}
$$

with $u_{0 j}=u_{i 0}=0$, then $m(I)$ is a measure on $\mathfrak{I}$, i.e., it is a countably additive extended positive valued set function defined on $\mathfrak{I}$ with $m(\varnothing)=0$. The factor $K$ is so chosen as to make $m(C)=1$. Let us denote this measure space by $(C, \mathfrak{J}, m)$.

As a measure on an interval class, $m$ can be extended to be a measure on a Borel field of sets in the usual way. Let $\mathcal{O}=\{\Omega\}$ where $\Omega$ is an $O$-set of $\mathfrak{I}$, i.e., it is the union of countably many members of $\mathfrak{I}$. The Carathéodory outer measure of an arbitrary set $\Gamma \subset C$ is defined to be

$$
m^{*}(\Gamma)=\inf _{\Omega \supset \Gamma} m(\Omega) .
$$

A set $\Gamma \subset C$ is called Carathéodory, and in our particular case Wiener, measurable if for every set $A \subset C$

$$
m^{*}(A)=m^{*}(A-\Gamma)+m^{*}(A \cap \Gamma) .
$$

The collection $\mathfrak{C}_{m}$ of Wiener measurable sets, the Carathéodory extension of $\mathfrak{I}$, is a Borel field and if we define $m(\Gamma)=m^{*}(\Gamma)$ for $\Gamma \in \mathfrak{C}_{m}, m$ is a Lebesgue measure on $\mathfrak{C}_{m}$. Let us denote this measure space by $\left(C, \mathfrak{C}_{m}, m\right)$.

The subset $\mathfrak{I}^{0}$ of $\mathfrak{I}$ consisting of intervals of the type

$$
\begin{aligned}
I^{0}\left\{x_{i}, y_{j}, \alpha_{i j}, \beta_{i j}, i=1,2, \cdots, m, j=1,2, \cdots, n\right\} \\
=\left\{f \in C ; \alpha_{i j}<f\left(x_{i}, y_{j}\right) \leqq \beta_{i j}, i=1,2, \cdots, m, j=1,2, \cdots, n\right\}
\end{aligned}
$$

where $-\infty \leqq \alpha_{i j}<\beta_{i j} \leqq+\infty$ is called the restricted interval class. It is an interval class by its own right and if we define $m^{0}(I)=m(I)$ for every $I^{0} \in \mathfrak{I}^{0}, m^{0}$ is a measure on $\mathfrak{I}^{0}$. If we now construct $\left(C, \mathfrak{C}_{m^{0}}, m^{0}\right)$ from $\left(C, \mathfrak{I}^{0}, m^{0}\right)$ the way we constructed $\left(C, \mathfrak{C}_{m}, m\right)$ from $(C, \mathfrak{I}, m)$, we have a Lebesgue measure $m^{0}$ on the Borel field $\mathfrak{C}_{m^{0}}$. In $\S 6$, Theorem IV, we show that $\left(C, \mathfrak{C}_{m^{0}}, m^{0}\right)=\left(C, \mathfrak{C}_{m}, m\right)$. This will enable us to express any $\Gamma \in \mathfrak{C}_{m}$ in terms of members of $\mathfrak{J}^{0}$.

Our main results are the following theorems:

THEOREM I. Let $H\left(u_{11}, \cdots, u_{m n}\right)$ be a Lebesgue measurable function of $m n$ real variables. Let $0<x_{1}<\cdots<x_{m} \leqq 1,0<y_{1}<\cdots<y_{n} \leqq 1$. Then the functional $H\left[f\left(x_{1}, y_{1}\right), \cdots, f\left(x_{m}, y_{n}\right)\right]$ defined on $C$ is Wiener measurable and

$$
\begin{aligned}
& \int_{C} H\left[f\left(x_{1}, y_{1}\right), \cdots, f\left(x_{m}, y_{n}\right)\right] d_{w} f \\
& =K\left\{\left[x_{i}\right],\left[y_{j}\right]\right\} \int_{-\infty}^{\infty}(m n) \int_{-\infty}^{\infty} H\left(u_{11}, \cdots, u_{m n}\right) W\left\{\left[x_{i}\right],\left[y_{j}\right],\left[u_{i j}\right]\right\} d u_{11}, \cdots, d u_{m n}
\end{aligned}
$$


where the existence of one side implies that of the other and the validity of the equality.

THEOREM II. Let $F[g]$ be a functional defined and Wiener integrable over $C$. Let $F[g]$ be bounded in every uniformly bounded subset of $C$ and let $F[g]$ be continuous in the sense that for any sequence $\left\{g^{(k)}\right\} \subset C$ which converges uniformly in the unit square $Q$ to $g \in C$

$$
\lim _{k \rightarrow \infty} F\left[g^{(k)}\right]=F[g]
$$

Let $f_{0} \in C$, let $\partial^{2} f_{0} / \partial y \partial x$ exist and be of bounded variation on $Q$, $\left(\partial^{2} / \partial y \partial x\right) f_{0}(0, y), \quad\left(\partial^{2} / \partial y \partial x\right) f_{0}(1, y), \quad\left(\partial^{2} / \partial y \partial x\right) f_{0}(x, 0), \quad\left(\partial^{2} / \partial y \partial x\right) f_{0}(x, 1)$ be of bounded variation on the respective unit intervals. Then under the translation

$$
g(x, y)=f(x, y)+f_{0}(x, y)
$$

the Wiener integral undergoes the transformation

$$
\text { (1.11) } \int_{C} F[g] d_{w} g=\exp \left\{-\int_{Q} \frac{\partial^{2} f_{0}}{\partial y \partial x} d^{2} f_{0}\right\} \int_{C} F\left[f+f_{0}\right] \exp \left\{-2 \int_{Q} \frac{\partial^{2} f_{0}}{\partial y \partial x} d^{2} f\right\} d_{w} f
$$

The Riemann-Stieltjes integrals appearing in Theorems II and III are defined in §3. In case $\partial^{2} f_{0} / \partial y \partial x$ is Riemann integrable on $Q, \int_{Q}\left(\partial^{2} f_{0} / \partial y \partial x\right) d^{2} f_{0}$ $=\iint_{Q}\left(\partial^{2} f_{0} / \partial y \partial x\right)^{2} d x d y$. Also $\partial^{2} f_{0} / \partial y \partial x$ may be replaced by $\partial^{2} f_{0} / \partial x \partial y$ throughout.

THEOREM 1II. Let $f_{0} \in C$ satisfy the conditions in Theorem II. Let $\Gamma \subset C$ be Wiener measurable and

$$
T \Gamma=\left\{f \in C ; f=g-f_{0}, g \in \Gamma\right\} .
$$

Then

$$
m(\Gamma)=\exp \left\{-\int_{Q} \frac{\partial^{2} f_{0}}{\partial y \partial x} d^{2} f_{0}\right\} \int_{T \Gamma} \exp \left\{-2 \int_{Q} \frac{\partial^{2} f_{0}}{\partial y \partial x} d^{2} f\right\} d_{w} f .
$$

Moreover if $F[g]$ is a Wiener measurable functional defined on $\Gamma$

$$
\int_{\Gamma} F[g] d_{w} g=\exp \left\{-\int_{Q} \frac{\partial^{2} f_{0}}{\partial y \partial x} d^{2} f_{0}\right\} \int_{T \Gamma} F\left[f+f_{0}\right] \exp \left\{-2 \int_{Q} \frac{\partial^{2} f_{0}}{\partial y \partial x} d f\right\} d_{w} f
$$

in the sense that the existence of one side implies that of the other and the validity of the equality.

2. Proof of Theorem I. Let $0<x_{1}<\cdots<x_{m} \leqq 1,0<y_{1}<\cdots<y_{n} \leqq 1$ be given and let $H\left(u_{11}, \cdots, u_{m n}\right)$ be a Lebesgue measurable function defined on $R_{m n}$. We begin with the case where $H$ is the characteristic function $\chi_{E}$ of a Lebesgue measurable set $E \subset R_{m n}$. Let $I$ be an interval in $C$ defined by (1.1) with our $\left\{x_{i}\right\}$, $\left\{y_{j}\right\}$ and $E$. Then $H\left[f\left(x_{1}, y_{1}\right), \cdots, f\left(x_{m}, y_{n}\right)\right]=\chi_{I}$ and 


$$
m(I)=\int_{I} d_{w} f=\int_{C} \chi_{I} d_{w} f=\int_{C} H\left[f\left(x_{1}, y_{1}\right), \cdots, f\left(x_{m}, y_{n}\right)\right] d_{w} f .
$$

On the other hand, according to (1.2)

$$
\begin{aligned}
m(I) & =K \int_{E}(m n) \int W d u_{11}, \cdots, d u_{m n}=K \int_{-\infty}^{\infty}(m n) \int_{-\infty}^{\infty} \chi_{E} W d u_{11}, \cdots, d u_{m n} \\
& =K_{1} \int_{-\infty}^{\infty}(m n) \int_{-\infty}^{\infty} H\left(u_{11}, \cdots, u_{m n}\right) W d u_{11}, \cdots, d u_{m n} .
\end{aligned}
$$

These two equations imply the validity of (1.8) for this special case.

The result now easily follows when $H$ is any finite linear combination of characteristic functions of disjoint measurable sets of $R_{m n}$.

If $H$ is a measurable extended positive valued function on $R_{m n}$, there exists a nondecreasing sequence of non-negative integrable simple functions $H_{k}$ which converges to $H$ at each point in $R_{m n}$. For each $H_{k},(1.8)$ holds. The limit may be passed under the integral sign by Levi's theorem on monotone convergence and (1.8) holds for $H$.

Finally when $H$ is a measurable extended positive valued function on $R_{m n}$, we define $H^{+}\left(u_{11}, \cdots, u_{m n}\right)=\max \left\{H\left(u_{11}, \cdots, u_{m n}\right), 0\right\}, H^{-}\left(u_{11}, \cdots, u_{m n}\right)$ $=\max \left\{-H\left(u_{11}, \cdots, u_{m n}\right), 0\right\}$, so that $H=H^{+}-H^{-}$. Then (1.8) holds for each of $H^{+}$and $H^{-}$. In case the integrals of $H^{+}$and $H^{-}$are not both infinite, the integral of $H$ exists and (1.8) holds.

3. Riemann-Stieltjes integral. We define Riemann-Stieltjes integrals of functions of $n$ variables and state a few theorems which can be proved more or less in the same way as in the one variable case.

Let $f\left(x^{1}, \cdots, x^{n}\right)$ be a function of $n$ real variables $x^{p}, 1 \leqq p \leqq n$. For each $p$ and prescribed values of $x^{p}$ and $\Delta x^{p}$, the operator $\Delta^{p}$ is defined by

$$
\Delta^{p} f=f\left(x^{1}, \cdots, x^{n}\right)-f\left(x^{1}, \cdots, x^{p-1}, x^{p}-\Delta x^{p}, x^{p+1}, \cdots, x^{n}\right) .
$$

Then for $p_{1} \neq p_{2}, 0 \leqq p_{1}, p_{2} \leqq n, \Delta^{p_{1}}$ and $\Delta^{p_{2}}$ commute, and

$$
\Delta^{p_{1}} \Delta^{p_{2}} \cdots \Delta^{p_{n}}=\Delta^{1} \Delta^{2} \cdots \Delta^{n}
$$

whenever $\left(p_{1}, p_{2}, \cdots, p_{n}\right)$ is a permutation of $(1,2, \cdots, n)$.

Let $f\left(x^{1}, \cdots, x^{n}\right)$ be defined on an $n$-dimensional interval $I=\prod_{p=1}^{n}\left\{x^{p} \mid a^{p} \leqq x^{p} \leqq b^{p}\right\}$. Let $\mathfrak{P}$ be a partition of $I$ into $\prod_{p=1}^{n} M_{p} n$-dimensional intervals by partition points satisfying $a^{p}=x_{0}^{p}<x_{1}^{p}<\cdots<x_{M_{-}}^{p}=b^{p}, p=1,2, \cdots, n$. Let $\Delta x_{i^{p}}^{p}=x_{i^{p}}^{p}-x_{i^{p}-1}^{p}$ and let $\Delta_{i^{p}}^{p}$ be the operator defined by (3.1) with the fixed values $x_{i^{p}}^{p}$ and $\Delta x_{i^{p}}^{p}$ for $i^{p}=1,2, \cdots, M_{p}$ and $p=1,2, \cdots, n$. Consider

$$
\sum_{i^{1}=1}^{M_{1}} \cdots \sum_{i^{n}=1}^{M_{n}}\left|\Delta_{i^{1}}^{1} \cdots \Delta_{i^{n}}^{n} f\right|
$$


Definition 1. If the collection of numbers (3.3) corresponding to the collection of all partitions $\{\mathfrak{P}\}$ of $I$ is bounded above we say that $f$ is of bounded variation on $I$, write $f \in \mathrm{B}$.V.(I), and call the least upper bound the total variation of $f$ on $I$ and write $V(f, I)$ for it.

Let $\xi_{i^{p}}^{p}$ satisfy $x_{i^{p}-1}^{p} \leqq \xi_{i^{p}}^{p} \leqq x_{i^{p}}^{p}$ for $i^{p}=1,2, \cdots, M_{p}$ and $p=1,2, \cdots, n$. Consider the Riemann-Stieltjes sum of $g\left(x^{1}, \cdots, x^{n}\right)$ with respect to $f\left(x^{1}, \cdots, x^{n}\right)$,

$$
S\{\mathfrak{P},\{\xi\}\}=\sum_{i^{1}=1}^{M_{1}} \ldots \sum_{i^{n}=1}^{M_{n}} g\left(\xi_{i^{1}}^{1}, \cdots, \xi_{i^{n}}^{n}\right) \Delta_{i^{1}}^{1} \cdots \Delta_{i^{n}}^{n} f .
$$

Let $|\mathfrak{P}|$, norm of $\mathfrak{P}$, be the greatest diagonal length of the $\sum_{p=1}^{n} M_{p}$ rectangles resulting from $\mathfrak{P}$.

Definition 2. We say that $g$ is Riemann-Stieltjes integrable with respect to on $I$ if there exists a number $J$ such that for every given $\varepsilon>0$ there corresponds some $\delta>0$ in such way that $|S\{\mathfrak{P},\{\xi\}\}-J|<\varepsilon$ whenever $|\mathfrak{P}|<\delta$. The number $J$ is called the Riemann-Stieltjes integral of $g$ with respect to $f$ on $I$ and is denoted by $\int_{I} g d^{n} f$.

THEOREM 1. A mean value theorem for $\Delta^{1} \Delta^{2} \cdots \Delta^{n} f$. Let $\Delta^{1} \Delta^{2} \cdots \Delta^{n} f$ be defined for some fixed values of $x^{p}$ and $\Delta x^{p}$ for $p=1,2, \cdots, n$. Let $\left(p_{1}, p_{2}, \cdots, p_{n}\right)$ be a permutation of $(1,2, \cdots, n)$. If the partial derivative $f_{p_{1} p_{2} \ldots p_{n}}$ exists on the open interval $\prod_{p=1}^{n}\left(x^{p}-\Delta x^{p}, x^{p}\right)$ then there exists a point $\left(\xi^{1}, \xi^{2}, \cdots, \xi^{p}\right)$ in the open interval such that

$$
\Delta^{1} \Delta^{2} \cdots \Delta^{n} f=f_{p_{1} p_{2} \ldots p_{n}}\left(\xi^{1}, \xi^{2}, \cdots, \xi^{n}\right) \Delta x^{1} \Delta x^{2} \cdots \Delta x^{n}
$$

Corollary. If $f$ is defined on a closed n-dimensional interval and if $f_{p_{1} p_{2} \cdots p_{n}}$ exists and is bounded in the interior of the interval then $f$ is of bounded variation on the interval.

THEOREM 2. AN EXISTENCE THEOREM. If $g$ is continuous and $f$ is of bounded variation on a closed $n$-dimensional interval I then $\int_{I} g d^{n} f$ exists.

ThEOREM 3. REDUCTION TO RIEMANN INTEGRAL. If $g\left(x^{1}, x^{2}, \cdots, x^{n}\right)$ is defined and $f_{p_{1} p_{2} \ldots p_{n}}$ where $\left(p_{1}, p_{2}, \cdots, p_{n}\right)$ is a permutation of $(1,2, \cdots, n)$ exists at every point

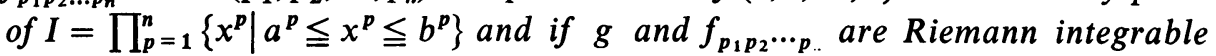
on $I$, then $g$ is Riemann-Stieltjes integrable with respect to $f$ there, and

$$
\int_{I} g d^{n} f=\int_{a^{1}}^{b^{1}} \cdots \int_{a^{n}}^{b^{n}} g f_{p_{1} p_{2} \ldots p_{n}} d x^{1} \cdots d x^{n} .
$$

This theorem is based on the following statement: If $\phi\left(x^{1}, \cdots, x^{n}\right)$ and $\psi\left(x^{1}, \cdots, x^{n}\right)$ are integrable on $I=\prod_{p=1}^{n}\left\{x^{p} \mid a^{p} \leqq x^{p} \leqq b^{p}\right\}$ then for every given $\varepsilon>0$ there exists some $\delta>0$ such that whenever $|\mathfrak{P}|<\delta$ and $x_{i p-1}^{p} \leqq \xi_{i p}^{p}$, $\eta_{i p}^{p} \leqq x_{i p}^{p}$ 
$\left|\sum_{i^{1}=1}^{M_{1}} \ldots \sum_{i^{n}=1}^{M_{n}} \phi\left(\xi_{i^{1}}^{1}, \cdots, \xi_{i^{n}}^{n}\right) \psi\left(\eta_{i^{1}}^{1}, \cdots, \eta_{i^{n}}^{n}\right) \Delta x_{i^{1}}^{1} \cdots \Delta x_{i^{n}}^{n}-\int_{a^{1}}^{b^{1}} \cdots \int_{a^{n}}^{b^{n}} \phi \psi d x^{1} \cdots d x^{n}\right|<\varepsilon$, where the integral in the inequality is the $n$-tuple Reimann integral of $\phi \psi$ on $I$.

4. A lemma on rearrangement of multiple series. We begin by defining a few operators. We use $\alpha\left(i^{1}, \cdots, i^{n}\right)$ rather than $\alpha_{i} \cdots_{i^{n}}$ to mean the term at the $\left(i^{1}, \cdots, i^{n}\right)$ position of an $n$-tuple series. Consider two such series $\alpha\left(i^{1}, \cdots, i^{n}\right)$ and $\beta\left(i^{1}, \cdots, i^{n}\right), i^{p}=0,1,2, \cdots, p=1,2, \cdots, n$.

Definition (1). For every integer $k, 0 \leqq k \leqq n$, and every subset $\left\{p_{1} p_{2}, \cdots, p_{k}\right\}$ of $\{1,2, \cdots, n\}$ the operator $D^{k}\left(p_{1}, \cdots, p_{k}\right)$ is defined by

with

$$
D^{k}\left(p_{1}, \cdots, p_{k}\right) \alpha\left(i^{1}, \cdots, i^{n}\right)=\alpha
$$

replaced by

$$
i^{p_{1}}, \cdots, i^{p_{k}}
$$

$$
i^{p_{1}}-1, \cdots, i^{p_{k}}-1
$$

$D^{k}\left(p_{1}, \cdots, p_{n}\right)$ operates on $\beta\left(i^{1}, \cdots, i^{n}\right)$ exactly in the same way as on $\alpha\left(i^{1}, \cdots, i^{n}\right)$. When there is no ambiguity we will write $D^{k}$ for $D^{k}\left(p_{1}, \cdots, p_{n}\right)$. For a given $k$, we use $\mathfrak{D}^{k}$ to mean the collection of all $D^{k}$ operators, $C_{n, k}$ in number.

Definition (2). For every integer $k, 0 \leqq k \leqq n$ and every division of $\{1,2, \cdots, n\}$ into three subsets, some possibly empty, $\left\{p_{1}, p_{2}, \cdots, p_{s}\right\},\left\{q_{1}, q_{2}, \cdots, q_{t}\right\}$ and $\left\{r_{1}, r_{2}, \cdots, r_{u}\right\}$ with $s+t=k, k+u=n$, the operator

$$
R^{k}\left(p_{1}, \cdots, p_{s}\left|q_{1}, \cdots, q_{t}\right| r_{1}, \cdots, r_{u}\right)
$$

or simply $R^{k}\left(p_{1}, \cdots, p_{s} \mid q_{1}, \cdots, q_{t}\right)$ or $R^{k}$ is defined with preassigned integers $M_{1}, \cdots, M_{n}$ by

with

$$
R^{k}\left(p_{1}, \cdots, p_{s} \mid q_{1}, \cdots, q_{t}\right) \alpha\left(i^{1}, \cdots, i^{n}\right)=\alpha
$$

replaced by 0 and

$$
i^{p_{1}}, \cdots, i^{p_{s}}
$$

replaced by

$$
i^{q_{1}}, \cdots, i^{q_{t}}
$$

and

$$
M_{q_{1}}, \cdots, M_{q_{t}}
$$

$$
R^{k}\left(p_{1}, \cdots, p_{s} \mid q_{1}, \cdots, q_{t}\right) \beta\left(i^{1}, \cdots, i^{n}\right)=\beta
$$

with

replaced by 0 and

$$
i^{p_{1}}, \cdots, i^{p_{*}}
$$

replaced by

$$
i^{q_{1}}, \cdots, i^{q_{t}}
$$

$$
M_{q_{1}}+1, \cdots, M_{q_{t}}+1
$$

For a given $k$ we use $\Re^{k}$ to denote the collection of $C_{n, k} 2^{k} R^{k}$ operators. 
DEFINITION (3). $\quad \sigma\left(R^{k}\left(p_{1}, \cdots, p_{s} \mid q_{1}, \cdots, q_{1}\right)\right)=t$.

LEMMA. With the notations defined above

$$
\begin{aligned}
& \sum_{i^{1}=1}^{M_{1}} \cdots \sum_{i^{n}=1}^{M_{n}} \beta\left(i^{1}, \cdots, i^{n}\right)\left[\sum_{k=0}^{n} \sum_{D^{k}}(-1)^{k} D^{k} \alpha\left(i^{1}, \cdots, i^{n}\right)\right] \\
&=\sum_{k=0}^{n} \sum_{\Re^{n-k}}(-1)^{n-\sigma\left(R^{n-k}\right)} \sum_{i^{r_{1}=0}}^{M_{r_{1}}} \ldots \sum_{i^{r_{k}=0}}^{M_{r_{k}}} R^{n-k} \alpha\left(i^{1}, \cdots, i_{n}\right) \\
& \cdot\left[\sum_{l=0}^{k} \sum_{D^{l}}(-1)^{l} D^{l} R^{n-k} \beta\left(i^{1}+1, \cdots, i^{n}+1\right)\right] .
\end{aligned}
$$

The proof is by straightforward induction and is left to the reader.

INTEGRATION BY PARTS. Consider the $n$-dimensional interval

$$
I^{n}=\prod_{p=1}^{n}\left\{x^{p} \mid a^{p} \leqq x^{p} \leqq b^{p}\right\}
$$

Let $0 \leqq k \leqq n$. If we choose $n-k$ of the $n$ variables and set each $x^{p}$ of this subcollection to be equal to $a^{p}$ or $b^{p}$ and let each $x^{p}$ of the remaining $k$ variables satisfy $a^{p} \leqq x^{p} \leqq b^{p}$ then we have a $k$-dimensional interval. Such an interval will be denoted by $I^{k}\left[x^{1}, \cdots, x^{n}\right]$ with the appropriate variables replaced by the appropriate constants $a^{p}$ or $b^{p}$. For fixed $k$ there are $C_{n, k} 2^{n-k}$ such intervals. Let the collection be denoted by $\mathfrak{I}^{k}$ and let $\mathfrak{I}=\bigcup_{k=0}^{n} \mathfrak{I}^{k}$. For each $I^{k} \in \mathfrak{I}$ let $\sigma\left(I^{k}\right)$ be the number of $b^{p}$ 's appearing in the square bracket which indicates the position of $I^{k}$.

Let $f$ and $g$ be defined on $I^{n}$. If $I^{k} \in \mathfrak{I}$ and the Riemann-Stieltjes integral of $f$ with respect to $g$ on $I^{k}$ exists we write $\int_{i^{k}} f d^{k} g$ for it. Hereby it is understood that if $I^{0} \in \mathfrak{I}$, then $\int_{I^{0}} f d^{0} g$ always exists and is the product of the values of $f$ and $g$ at that point which enters in $\mathfrak{I}$ as the 0 -dimensional interval $I^{0}$.

THEOREM 4. Let $f$ be Riemann-Stieltjes integrable with respect to $g$ on each member of $\mathfrak{I}$, then $g$ is Riemann-Stieltjes integrable with respect to $f$ and

$$
\int_{I^{n}} g d^{n} f=\sum_{k=0}^{n} \sum_{\mathfrak{J}^{k}}(-1)^{n-\sigma\left(I^{k}\right)} \int_{I^{k}} f d^{k} g .
$$

Proof. Consider a partition of $I^{n}$ defined by $a^{p}=x_{1}^{p}<x_{2}^{p}<\cdots<x_{M_{p}}^{p}=b^{p}$ for $p=1,2, \cdots, n$ and let $x_{i^{p}}^{p} \leqq \xi_{i^{p}}^{p_{1}} \leqq x_{i^{p}}^{p}, i^{p}=1,2, \cdots, M_{p}$, and $\xi_{0}^{p}=a^{p}, \xi_{M_{p}+1}^{p}=b^{p}$ for $p=1,2, \cdots, n$. Let $\alpha\left(i^{1}, \cdots, i^{n}\right)=f\left(x_{i^{1}}^{1}, \cdots, x_{i^{n}}^{n}\right)$ and $\beta\left(i^{1}, \cdots, i^{n}\right)=g\left(\xi_{i 1}^{1}, \cdots, \xi_{i^{n}}^{n}\right)$. Then the Riemann-Stieltjes sum of $g$ with respect to $f$ and $I^{n}$ becomes the left side of (4.1). To each $R^{n-k}$ in the right side of (4.1) there corresponds in the obvious way an $I^{k} \in \mathfrak{I}$. Furthermore,

$$
\sum_{i^{r_{1}}=0}^{M_{r_{1}}} \cdots \sum_{i^{r_{k}=0}}^{M_{r_{k}}} R^{n-k} \alpha\left(i^{1}, \cdots, i^{n}\right) \sum_{l=0}^{k} \sum_{D^{l}}(-1)^{l} D^{l} R^{n-k} \beta\left(i^{1}+1, \cdots, i^{n}+1\right)
$$


is the Riemann-Stieltjes sum of $f$ with respect to $g$ on $I^{k}$ corresponding to $\mathfrak{P}$ and $\{\xi\}$. It is obvious that $\sigma\left(R^{n-k}\right)=\sigma\left(I^{k}\right)$. From the existence of $\int_{I^{k}} f d^{k} g$ for every $I^{k} \in \mathfrak{I}$, (4.1) becomes (4.2) as $|\mathfrak{P}| \rightarrow 0$.

5. Definition. Let $n$ be a positive integer and consider the division of $Q$ into $n^{2}$ squares by means of the division points $x_{i}=i / n, y_{j}=j / n, i, j=0,1,2, \cdots, n$ of the unit intervals. Divide each square with corners $\left(x_{i-1}, y_{j-1}\right),\left(x_{i}, y_{j-1}\right)$, $\left(x_{i}, y_{j}\right),\left(x_{i-1}, y_{j}\right)$ in two rectangular triangles by the diagonal with the endpoints $\left(x_{i-1}, y_{j-1}\right)$ and $\left(x_{i}, y_{j}\right)$. For $f \in C, L_{n}[f]$ is defined to be equal to $f(x, y)$ at each $\left(x_{i}, y_{j}\right), i, j=0,1,2, \cdots, n$ and linear on each of the $2 n^{2}$ rectangular triangles. In other words if $(x, y)$ is in the triangle with $\left(x_{i-1}, y_{j-1}\right),\left(x_{i}, y_{j-1}\right),\left(x_{i}, y_{j}\right)$ as corners

$$
\begin{aligned}
L_{n} f(x, y)=\frac{1}{n}\{ & \left.f\left(\frac{i}{n}, \frac{j-1}{n}\right)-f\left(\frac{i-1}{n}, \frac{j-1}{n}\right)\right\}\left(x-\frac{i}{n}\right) \\
& +\frac{1}{n}\left\{f\left(\frac{i}{n}, \frac{j}{n}\right)-f\left(\frac{i}{n}, \frac{j-1}{n}\right)\right\}\left(y-\frac{j-1}{n}\right)+f\left(\frac{i}{n}, \frac{j-1}{n}\right)
\end{aligned}
$$

and if $(x, y)$ is in the triangle with $\left(x_{i-1}, y_{j-1}\right),\left(x_{i-1}, y_{j}\right),\left(x_{i}, y_{j}\right)$ as corners

$$
\begin{aligned}
L_{n} f(x, y)=\frac{1}{n}\{f & \left.\left(\frac{i}{n}, \frac{j}{n}\right)-f\left(\frac{i-1}{n}, \frac{j}{n}\right)\right\}\left(x-\frac{i-1}{n}\right) \\
& +\frac{1}{n}\left\{f\left(\frac{i-1}{n}, \frac{j}{n}\right)-f\left(\frac{i-1}{n}, \frac{j-1}{n}\right)\right\}\left(y-\frac{j}{n}\right)+f\left(\frac{i-1}{n}, \frac{j}{n}\right) .
\end{aligned}
$$

Lemma 1. Let $F[f]$ be a functional defined on $C$. Let $F[f]$ be continuous in the sense that $\lim _{k \rightarrow \infty} F\left[f^{(k)}\right]=F\left[f^{(0)}\right]$ whenever $\left\{f^{(k)}\right\} \subset C$ converges uniformly on $Q$ to $f^{0} \in C$. Then the functional $F\left[L_{n}[f]\right]$ defined on $C$ is Wiener measurable and there exists a continuous real-valued function $H\left(u_{11}, \cdots, u_{n n}\right)$ defined on $R_{n n}$ such that

$$
F\left[L_{n}[f]\right]=H\left[f\left(x_{1}, y_{1}\right), \cdots, f\left(x_{n}, y_{n}\right)\right] .
$$

Proof. Since $L_{n}[f]$ depends only on the $n^{2}$ values of $f$ at $\left(x_{i}, y_{j}\right)$, there exists a real-valued function $H$ for which (5.3) holds.

To show the continuity of $H$, let $\lim _{k \rightarrow \infty} u^{(k)}=u^{(0)}$ where

$$
u^{(k)}=\left[u_{11}^{(k)}, \cdots, u_{n n}^{(k)}\right] \text { for } k=0,1,2, \cdots .
$$

Let $f^{(k)} \in C, k=0,1,2, \cdots$, be linear on each of the $2 n^{2}$ triangles in the definition of $L_{n}$ and satisfy

$$
f^{(k)}\left(x_{i}, y_{j}\right)=u_{i j}^{(k)}, \quad k=0,1,2, \cdots .
$$

Since $\lim _{k \rightarrow \infty} u^{(k)}=u^{(0)}$, we have $\lim _{k \rightarrow \infty} f^{(k)}(x, y)=f^{(0)}(x, y)$ uniformly on $Q$ and hence $\lim _{k \rightarrow \infty} F\left[f^{(k)}\right]=f\left[F^{(0)}\right]$. Now $L_{n}\left[f^{(k)}\right]=f^{(k)}$ for $k=0,1,2, \cdots$ and 


$$
F\left[f^{(k)}\right]=F\left[L_{n}\left[f^{(k)}\right]\right]=H\left[f^{(k)}\left(x_{1}, y_{1}\right), \cdots, f^{(k)}\left(x_{n}, y_{n}\right)\right]=H\left[u_{11}^{(k)}, \cdots, u_{n n}^{(k)}\right]
$$

for $k=0,1,2, \cdots$. Thus $\lim _{k \rightarrow \infty} H\left(u^{(k)}\right)=H\left(u^{(0)}\right)$ and $H$ is continuous.

To prove the Wiener measurability of $F\left[L_{n}[f]\right]$, we note that for any real number $\alpha$

$$
\begin{aligned}
\Gamma_{\alpha} & =\left\{f \in C ; F\left[L_{n}[f]\right]>\alpha\right\} \\
& =\left\{f \in C ; H\left[f\left(x_{1}, y_{1}\right), \cdots, f\left(x_{n}, y_{n}\right)\right]>\alpha\right\} \\
& =\left\{f \in C ;\left[f\left(x_{1}, y_{1}\right), \cdots, f\left(x_{n}, y_{n}\right)\right] \in E\right\}
\end{aligned}
$$

where $E=\left\{u \in R_{n n} ; H(u)>\alpha\right\}$ which is a Lebesgue measurable set since $H(u)$ is continuous and measurable. Thus $\Gamma_{\alpha} \in \mathfrak{I}$ and is Wiener measurable. The arbitrariness of $\alpha$ implies the measurability of $F\left[L_{n}[f]\right]$.

Lemma 2. Let $f_{0}, f \in C$ and let $f_{0}$ satisfy the conditions in Theorem II. Let $\mathfrak{P}$ be a partition of $Q$ by $0=x_{0}<x_{1}<\cdots<x_{m}=1,0=y_{0}<y_{1}<\cdots<y_{n}=1$, and let

$$
\Delta_{i} \Delta_{j} f=f\left(x_{i}, y_{j}\right)-f\left(x_{i-1}, y_{j}\right)-f\left(x_{i}, y_{j-1}\right)+f\left(x_{i-1}, y_{j-1}\right)
$$

then

$$
\lim _{|\mathfrak{B}| \rightarrow 0} \sum_{i=1}^{m} \sum_{j=1}^{n}\left\{\frac{\left(\Delta_{i} \Delta_{j} f_{0}\right)^{2}}{\Delta_{i} x \Delta_{j} y}+\frac{2\left(\Delta_{i} \Delta_{j} f_{0}\right)\left(\Delta_{i} \Delta_{j} f\right)}{\Delta_{i} x \Delta_{j} y}\right\}=\int_{Q} \frac{\partial^{2} f_{0}}{\partial y \partial x} d^{2} f_{0}+2 \int_{Q} \frac{\partial^{2} f_{0}}{\partial y \partial x} d^{2} f,
$$

where the convergence is bounded in $f$ for all $f$ in any uniformly bounded set. In case $\partial^{2} f_{0} / \partial y \partial x$ is Riemann integrable on $Q, \int_{Q}\left(\partial^{2} f_{0} / \partial y \partial x\right) d^{2} f_{0}$ reduces to $\iint_{Q}\left(\partial^{2} f_{0} / \partial y \partial x\right)^{2} d x d y$.

Proof. By Theorem 1.

$$
D_{i j}[f, \mathfrak{P}]=\frac{\left(\Delta_{i} \Delta_{j} f_{0}\right)^{2}}{\Delta_{i} x \Delta_{j} y}+\frac{2\left(\Delta_{i} \Delta_{j} f_{0}\right)\left(\Delta_{i} \Delta_{j} f\right)}{\Delta_{i} x \Delta_{j} y}=\frac{\partial^{2}}{\partial y \partial x} f_{0}\left(\xi_{i}, \eta_{j}\right)\left\{\Delta_{i} \Delta_{j} f_{0}+2 \Delta_{i} \Delta_{j} f\right\},
$$

where $x_{i-1}<\xi_{i}<x_{i}, y_{j-1}<\eta_{j}<y_{j}$. Since $\partial^{2} f_{0} / \partial y \partial x$ is of bounded variation on $Q_{0}, f_{0}, f$ are continuous on $Q$ and $\left(\partial^{2} / \partial y \partial x\right) f_{0}(0, y),\left(\partial^{2} / \partial y \partial x\right) f_{0}(1, y)$, $\left(\partial^{2} / \partial y \partial x\right) f_{0}(x, 0),\left(\partial^{2} / \partial y \partial x\right) f_{0}(x, 1)$ are of bounded variation on the respective unit intervals, Theorems 3 and 4 apply and

$$
\lim _{|\mathfrak{B}| \rightarrow 0} \sum_{i=1}^{m} \sum_{j=1}^{n} D_{i j}[f, \mathfrak{P}]=\int_{Q} \frac{\partial^{2} f_{0}}{\partial y \partial x} d^{2} f_{0}+2 \int_{Q} \frac{\partial^{2} f_{0}}{\partial y \partial x} d^{2} f
$$

For an arbitrary $M>0$, let $\Gamma=\{f \in C ;|f(x, y)| \leqq M$ on $Q\}$. It follows easily from the lemma of $\S 4$ that $\sum D_{i j}[f, p]$ is bounded on $\Gamma$.

Proof of Theorem II. Let $M$ be a positive number and $n$ be a positive integer. Define 
418

$$
\begin{aligned}
& C_{M}=\{g \in C ;|g(x, y)| \leqq M \text { on } Q\} \\
& C_{M, n}=\left\{g \in C ;\left|g\left(\frac{i}{n}, \frac{j}{n}\right)\right| \leqq M, i, j=0,1,2, \cdots, n\right\} .
\end{aligned}
$$

Then $C_{M, n} \supset C_{M, 2}{ }^{n}, \lim _{n \rightarrow \infty} C_{M, 2}{ }^{n}=C_{M}, C_{M} \subset C_{M^{\prime}}$ if $M<M^{\prime}$ and $\lim _{M \rightarrow \infty} C_{M}=C$. Let

$$
T C_{M}=\left\{f \in C ; f=g-f_{0}, g \in C_{M}\right\}
$$

By (5.3), (1.8)

$$
\begin{aligned}
\int_{C_{M, n}} F\left[L_{n}[g]\right] & d_{w} g \\
& =\int_{C_{M, n}} H\left[g\left(x_{1}, y_{1}\right), \cdots, g\left(x_{n}, y_{n}\right)\right] d_{w} g \\
& =K\left\{\left[x_{i}\right],\left[y_{j}\right]\right\} \int_{-M}^{M}(n n) \int_{-M}^{M} H\left[\left[v_{i j}\right]\right] W\left\{\left[x_{i}\right],\left[y_{j}\right],\left[v_{i j}\right]\right\} d v_{11} \cdots d v_{n n} .
\end{aligned}
$$

Since $L_{n}[g]=L_{n}[f]+L_{n}\left[f_{0}\right]$, if we write

$$
\begin{aligned}
v_{i j} & =g\left(x_{i}, y_{j}\right), \\
u_{i j} & =f\left(x_{i}, y_{j}\right), \\
a_{i j} & =f_{0}\left(x_{i}, y_{j}\right), \\
\Delta_{i} \Delta_{j} u & =u_{i j}-u_{i-1, j}-u_{i, j-1}+u_{i-1, j-1}
\end{aligned}
$$

and similarly for $\Delta_{i} \Delta_{j} a$, and apply (1.8),

$$
\begin{gathered}
=K\left\{\left[x_{i}\right],\left[y_{j}\right]\right\} \int_{-M-a_{11}}^{M-a_{11}}(n n) \int_{-M-a_{n n}}^{M-a_{n}} H\left[\left[u_{i j}+a_{i j}\right]\right] W\left\{\left[x_{i}\right],\left[y_{j}\right],\left[u_{i j}+a_{i j}\right]\right\} d u_{11} \cdots d u_{n n} \\
=\exp \left\{-\sum_{i=1}^{n} \sum_{j=1}^{n} \frac{\left(\Delta_{i} \Delta_{j} a\right)^{2}}{\Delta_{i} x \Delta_{j} y}\right\} \int_{-M-a_{11}}^{M-a_{11}}(n n) \int_{M-a_{n n}}^{M+a_{n n}} H\left[\left[u_{i j}+a_{i j}\right]\right] \\
\cdot \exp \left\{-\sum_{i=1}^{n} \sum_{j=1}^{n} \frac{\left(\Delta_{i} \Delta_{j} u\right)^{2}}{\Delta_{i} x \Delta_{j} y}\right\} \\
\cdot \exp \left\{-\sum_{i=1}^{n} \sum_{j=1}^{n} \frac{2\left(\Delta_{i} \Delta_{j} a\right)\left(\Delta_{i} \Delta_{j} u\right)}{\Delta_{i} x \Delta_{j} y}\right\} d u_{11} \cdots d u_{n n} \\
=\exp \left\{-\sum_{i=1}^{n} \sum_{j=1}^{n} \frac{\left(\Delta_{i} \Delta_{j} f_{0}\right)^{2}}{\Delta_{i} x \Delta_{j} y}\right\} \int_{T C_{M, n}} F\left[L_{n}\left[f+f_{0}\right]\right] \exp \left\{-2 \sum_{i=1}^{n} \sum_{j=1}^{n} \frac{\left(\Delta_{i} \Delta_{j} f_{0}\right)\left(\Delta_{i} \Delta_{j} f\right)}{\Delta_{i} x \Delta_{i} y}\right\} d_{w} f
\end{gathered}
$$


Equation (5.4) is valid for any $C_{M, n}$, and in particular for every one of the sequence $\left\{C_{M, 2^{n}}\right\}, n=1,2, \cdots$.

Now for any $g \in C, \lim _{n \rightarrow \infty} L_{n}[g]=g$ uniformly on $Q$. This follows from (5.1), (5.2) and the fact that for any $\varepsilon>0$, there exists a positive integer $n$ such that $\left|g\left(x^{\prime}, y^{\prime}\right)-g\left(x^{\prime \prime}, y^{\prime \prime}\right)\right|<\varepsilon / 3$ whenever $\left|x^{\prime}-x^{\prime \prime}\right|<1 / n$ and $\left|y^{\prime}-y^{\prime \prime}\right|<1 / n$.

If we let $n \rightarrow \infty$ in (5.4) over the sequence of sets $\left\{C_{M, 2^{n}}\right\}$, then from the boundedness of $F$ and the sum under integral sign in the right side of (5.4), the limiting process may be passed under the integral signs. By Lemma 2 and the continuity of $F$, we have

$$
\int_{C_{M}} F[g] d_{w} g=\exp \left\{-\int_{Q} \frac{\partial^{2} f_{0}}{\partial y \partial x} d^{2} f_{0}\right\} \int_{C_{M}} F\left[f+f_{0}\right] \exp \left\{-2 \int_{Q} \frac{\partial^{2} f_{0}}{\partial y \partial x} d^{2} f\right\} d_{w} f .
$$

If we let $M$ approach infinity, we have (1.10).

6. Theorem IV. $\left(C, \mathfrak{C}_{m 0}, m^{0}\right)=\left(C, \mathfrak{C}_{m}, m\right)$.

Lemma 3. Let $\mathfrak{D}^{0}=\left\{\Omega^{0}\right\}$ be the family of $O$-sets of $\mathfrak{I}^{0}$. For every $\Gamma \subset C$, let

$$
m^{0 *}(\Gamma)=\inf _{\Omega^{0} \supseteq \Gamma} m^{0}\left(\Omega^{0}\right)
$$

Then $m^{0 *}(\Gamma)=m^{*}(\Gamma)$.

The proof is left to the reader.

Lemma 4. $C_{m^{0}}{ }^{*}=C_{m^{*}}$

This lemma is an obvious consequence of Carathéodory's theory of measure.

Proof of Theorem IV. It is well known that for any $\sigma$-finite measure on an interval class, there exists uniquely a countably additive extension of the measure to the Carathéodory extension of the interval class. Now since $m^{0}$ on $\mathbb{C}_{m}{ }^{0}$ and $m$ on $\mathfrak{C}_{m}$ are both a countably additive extension of $m$ on $\mathfrak{J}^{0}$ to $\mathfrak{C}_{m}{ }^{0}=\mathfrak{C}_{m}$, we have $m^{0}=m$ on $\mathfrak{C}_{m}^{0}=\mathfrak{C}_{m}$.

7. Definition. By $\mathfrak{D}^{0 \downarrow}$ we mean the class of limits of decreasing sequences of members of $\mathfrak{D}^{0}$.

Lemma 5. If $\Gamma \subset C$ is Wiener measurable, we can write $\Gamma=G-N$ where $G \in \mathbb{D}^{0 \downarrow}$ and $N \subset G$ with $m(N)=0$.

Proof. Let $\Gamma$ be Wiener measurable. Since $\left(C, \mathfrak{C}_{m}, m\right)=\left(C, \mathfrak{C}_{m}{ }^{0}, m^{0}\right)$, there exists some $\Omega_{n}^{0} \in \mathfrak{D}^{0}$ such that $\Omega_{n}^{0} \supset \Gamma$ and $m\left(\Omega_{n}^{0}-\Gamma\right)<1 / n$ for any positive integer. Let $G_{n}=\bigcap_{k=1} \Omega_{k}$. Then $G_{n} \in \mathcal{D}^{0}$. For if we let $\Omega_{1}^{0}=\bigcup_{k=1}^{\infty} I_{1, k}^{0}, \Omega_{2}^{0}=\bigcup_{l=1}^{\infty} I_{2}^{0}$, where $I_{1, k}^{0}, I_{2, l}^{0} \in \mathfrak{I}^{0}$,

$$
\Omega_{1}^{0} \cap \Omega_{2}^{0}=\left(\bigcup_{k=1}^{\infty} I_{1, k}^{0}\right) \cap\left(\bigcup_{l=1}^{\infty} I_{2, l}^{0}\right)=\bigcup_{k=1}^{\infty} \bigcup_{l=1}^{\infty}\left(I_{1, k}^{0} \cap I_{2, l}^{0}\right) \in \mathbb{D}^{0}
$$


because $I_{1, k}^{0} \cap I_{2, l}^{0} \in \mathfrak{I}^{0}$. By repetition we see that $G_{n} \in \mathfrak{D}^{0}$ for all $n$. Also $G_{n} \supset G_{n+1}, G_{n} \supset \Gamma$ for all $n$. Thus if we let $G=\lim _{n \rightarrow \infty} G_{n}$, then $G \in \mathfrak{D}^{0 \downarrow}, G \supset \Gamma$ and

$$
m(G-\Gamma) \leqq m\left(G_{n}-\Gamma\right) \leqq m\left(\Omega_{n}^{0}-\Gamma\right)<\frac{1}{n} .
$$

If we let $n$ tend to infinity, we have $m(G-\Gamma)=0$. Let $N=G-\Gamma$. Then $\Gamma=G-N, N \subset G$ and $m(N)=0$.

Proof of Theorem III. To prove (1.13) we consider first the case where $\Gamma=I^{0}$ as given by (1.7). Let $\phi_{i j, k}(u)$ be a continuous trapezoidal function which equals one on $\left[\alpha_{i j}+1 / k, \beta_{i j}\right]$, equals zero on $\left(-\infty, \alpha_{i j}\right]$ and $\left[\beta_{i j}+1 / k,+\infty\right)$ and is linear on $\left[\alpha_{i j}, \alpha_{i j}+1 / k\right]$ and $\left[\beta_{i j}, \beta_{i j}+1 / k\right]$. If $\alpha_{i j}, \beta_{i j}$ are infinite so are $\alpha_{i j}+1 / k, \beta_{i j}+1 / k$. Let

$$
\chi_{I^{0}, k}[g]=\prod_{i=1}^{m} \prod_{j=1}^{n} \phi_{i j, k}\left[g\left(x_{i}, y_{j}\right)\right], \quad g \in C .
$$

Each $\phi_{i j, k}\left[g\left(x_{i}, y_{j}\right)\right]$ is Wiener measurable by Theorem $I$ and so is $\chi_{1^{0}, k}[g]$. Since $0 \leqq \chi_{I^{0}, k}[g] \leqq 1, \chi_{I^{0}, k}[g]$ is uniformly bounded in $C$. If $\left\{g^{(n)}\right\} \subset C$ and $\lim _{n \rightarrow \infty} g^{(n)}(x, y)=g(x, y)$ on $Q$, the continuity of $\phi_{i j, k}[u]$ implies

$$
\lim _{n \rightarrow \infty} \phi_{i j, k}\left[g^{(n)}\left(x_{i}, y_{j}\right)\right]=\phi_{i j, k}\left[g\left(x_{i}, y_{j}\right)\right]
$$

and

$$
\lim _{n \rightarrow \infty} \chi_{I^{0}, k}\left[g^{(n)}\right]=\chi_{I^{0}, k}[g]
$$

Thus $\chi_{I^{0}, k}[g]$ satisfies the conditions on $F$ in Theorem II and (1.10) holds with $F[g]=\chi_{I^{0}, k}[g]$. Since $\lim _{k \rightarrow \infty} \chi_{I^{0}, k}[g]=\chi_{I^{0}}[g]$ monotonically, (1.10) holds wih $F[g]=\chi_{I}[g]$, which is the validity of (1.13) when $\Gamma=I^{0}$. The equality (1.13) holds when $\Gamma=\Omega^{0} \in \mathfrak{D}^{0}$, since both sides of it are completely additive functions of $\Gamma$. Finally when $\Gamma$ is an arbitrary measurable set, it can be written as $\Gamma=G-N$ where $G \in \mathfrak{D}^{0 \downarrow}, N \subset G, m(N)=0$ according to Lemma 5 so that by monotone convergence, (1.13) holds.

The equality (1.14) can be proved exactly in the same way as in the one variable case.

\section{BIBLIOGRAPHY}

1. R. H. Cameron and W. T. Martin, Transformations of Wiener integrals under translations, Ann. of Math. (2) 45 (1944), 386-396.

2. T. Kitagawa, Analysis of variance applied to function spaces, Mem. Fac. Sci. Kyushu Univ. Ser. A 6 (1951), 41-53.

3. J. Yeh, Wiener measure in a space of functions of two variables, Trans. Amer. Math. Soc. 95 (1960), 433-450.

4. V. I. Smirnov, Kurs Vysshei matematiki (A course in higher mathamatics), Vol. 5, Fizmatgiz, Moscow, 1960 (Russian).

5. C. Carathéodory, Vorlesungen über reele Funktionen, Teubner, Leipzig, 1927.

UNIVERSITY OF ROCHESTER,

ROCHESTER, NEW YORK 